\title{
BMJ Open Bridging the gap between physical and mental illness in community pharmacy (PharMIbridge): protocol for an Australian cluster randomised controlled trial
}

\author{
Amanda J Wheeler (D) , ${ }^{1,2}$ Claire L O'Reilly (D) , ${ }^{3}$ Sarira El-Den (D) , ${ }^{3}$ Joshua Byrnes, ${ }^{1}$ \\ Robert S Ware, ${ }^{1}$ Sara S McMillan (D) ${ }^{1,4}$
}

To cite: Wheeler AJ, O'Reilly CL, El-Den S, et al. Bridging the gap between physical and mental illness in community pharmacy (PharMlbridge): protocol for an Australian cluster randomised controlled trial. BMJ Open 2020;10:e039983. doi:10.1136/ bmjopen-2020-039983

- Prepublication history for this paper is available online. To view these files, please visit the journal online (http://dx.doi. org/10.1136/bmjopen-2020039983).

AJW and CLO are joint first authors.

Received 01 May 2020

Revised 20 May 2020

Accepted 21 May 2020

Check for updates

(C) Author(s) (or their employer(s)) 2020. Re-use permitted under CC BY-NC. No commercial re-use. See rights and permissions. Published by BMJ.

For numbered affiliations see end of article.

Correspondence to Professor Amanda J Wheeler; a.wheeler@griffith.edu.au

\section{ABSTRACT}

Introduction There is a significant life expectancy gap attributable to physical comorbidities for people living with severe and persistent mental illness (SPMI) compared with the general population. Medications are a major treatment for SPMI management and physical illnesses, hence pharmacists are well positioned to support mental healthcare and comorbidities. The randomised controlled trial (RCT) aim is to evaluate effectiveness of an individualised, pharmacist led, support service for people experiencing SPMI focusing on medication adherence and physical comorbidity management, compared with standard care (a medication-management service; MedsCheck).

Methods and analysis PharMlbridge is a cluster RCT, whereby community pharmacies in four Australian regions will be randomised (1:1 ratio), to either Intervention Group (IG) or Comparator Group (CG). All IG and CG pharmacy staff will receive Blended-Mental Health First Aid training. Additionally, IG pharmacists will receive further training on medication adherence, goal setting, motivational interviewing, managing physical health concerns and complex issues relating to psychotropic medication. CG pharmacists will not receive additional training, and will provide standard care (MedsCheck). The primary outcome will be change in participants medication adherence for psychotropic medication over 6-months. Using mixedeffects logistic regression model and a cluster size of 48 pharmacies, a total of 190 participants will need to be recruited to each arm to find a statistically significant difference in medication adherence. Secondary outcomes will be changes in factors associated with cardiometabolic risk and quality of life, emphasising physical and psychological well-being; medication-related problems; adherence to other prescribed medication; pharmacists knowledge, confidence and ability to support people experiencing SPMI; and effects on healthcare utilisation. A within RCT-based economic evaluation comparing the intervention with standard care will be undertaken. Ethics and dissemination The protocol and pharmacist training programme received Griffith University Human Research Ethics Committee approval (HREC/2019/473 and HREC/2019/493 respectively). Results will be published
Strengths and limitations of this study

- The PharMIbridge randomised controlled trial (RCT) builds on previous Australian research to test the effectiveness of a pharmacist-led medication support service for consumers living with severe and persistent mental illness (SPMI), with an additional focus on the management of physical comorbidities which are highly prevalent in this population.

- A multidisciplinary approach will encourage participating pharmacies to develop and maintain working relationships with local general practitioner clinics, medical centres, mental healthcare teams, and other physical health/exercise centres and practitioners (eg, gyms) beyond the life of the RCT.

- Routinely collected pharmacy dispensing and Pharmaceutical Benefits Scheme (PBS) data will be used, alongside self-reported measurements, to evaluate medication adherence, which is the primary outcome measure.

- While a key focus of this RCT is exploring the impact of the PharMlbridge intervention on the physical well-being and overall health of people living with SPMI, the follow-up time is insufficient to use these and related outcomes as primary outcome measures.

- It is not possible for pharmacy staff or consumer participants to be blind to service allocation, due to the nature of the intervention.

in peer-reviewed journals and available at the Sixth Community Pharmacy Agreement website (http://6cpa. com.au/about-6cpa/).

Trial registration number ANZCTR12620000577910.

\section{BACKGROUND AND RATIONALE}

Community pharmacists are highly trusted and essential members of the primary healthcare team, playing a vital role through medication supply and information provision. Given the accessibility of community pharmacies, 
pharmacists are in an ideal position to adopt a triagestyle role, signposting to other healthcare professionals and services. There is an emerging role and growing need, for community pharmacists to support and manage mental illnesses. Research has demonstrated that pharmacists are capable of identifying consumers at risk of depression and referring appropriately for diagnosis and therapy, ${ }^{1-3}$ managing psychotropic (eg, antipsychotic and mood stabiliser) medication-related problems (MRPs), ${ }^{4-7}$ providing antidepressant adherence support and working within multidisciplinary mental healthcare teams. ${ }^{8-10}$

However, evidence also suggests that healthcare professionals, including pharmacists, are not exempt from holding stigmatising attitudes and behaviours towards mental health consumers; ${ }^{11}{ }^{12}$ pharmacists with more marked stigmatising attitudes were reported to be less willing to provide professional services to people with severe and persistent mental illness ${ }^{\mathrm{i}}$ (SPMI) ${ }^{13}$ Lack of training in mental health or the opportunity to acquire the necessary skills and confidence in working with mental health consumers have been cited as barriers to providing appropriate and timely care for this population. ${ }^{14}$ Hence, the urgent need to develop a training programme that addresses pharmacists' mental health knowledge gaps in anticipation of improving attitudes towards, and confidence in, providing care for people living with SPMI. This is particularly important in the Australian context given that $61.5 \%$ of people who experience psychosis, do so on multiple occasions, with partial or complete remission of symptoms between episodes. ${ }^{15}$ Also of relevance is that a substantial proportion of Australians using psychotropic medications access healthcare services in community settings, with 39 million mental health-related prescriptions dispensed in Australia in 2018-2019. ${ }^{16}$

Of significant concern is that Australians living with SPMI also experience poor physical health; they are five times more likely to smoke, six times more likely to die from cardiovascular disease and four times more likely to die from respiratory disease than the general population, leading to a 14-23years gap in life expectancy. ${ }^{17}$ These issues are often overlooked and/or dismissed by healthcare professionals and policy-makers. ${ }^{18}$ For example, a study by Morant $e t a l^{19}$ in the UK found that the physical health concerns of antipsychotic users were not addressed by prescribing psychiatrists. Decisive action is needed to engage both relevant health practitioner groups and people living with SPMI with respect to physical health management. ${ }^{20}$

The acceptability of medications by people living with SPMI is also problematic and adherence is poor. ${ }^{21}$ Reasons include, but are not limited to, experiencing (or the risk of experiencing) unwanted side effects, reluctance to rely on medication and forgetfulness. ${ }^{21}$

${ }^{\mathrm{i}}$ The World Health Organization defines severe mental illness as '...a group of conditions that include moderate to severe depression, bipolar disorder, and schizophrenia and other psychotic disorders. ${ }^{78}$
Poor adherence with antipsychotics is a key reason for relapse in psychosis; even missing a few days of treatment substantially increases this risk. ${ }^{22}$ However, a good therapeutic relationship with attending health practitioners is linked to better medication adherence, fewer hospital admissions and a reduction in self-harm in people experiencing psychosis. ${ }^{23}$ In this respect, pharmacist-led medication reviews for mental health consumers have shown promising results, ${ }^{724}$ and given the associated concerns with drug-drug interactions, are particularly relevant for those living with SPMI using psychotropic medications.

\section{Evidence gap}

Overall, there is relatively limited research on improving outcomes for consumers living with SPMI in the primary care setting (including community pharmacy). While research exploring the impact of medication reviews on psychotropic prescribing within residential aged care settings has been conducted, ${ }^{4}$ few studies have involved participants using psychotropic medications living in the community, ${ }^{25}{ }^{26}$ particularly those experiencing SPMI. ${ }^{27}$ While Australian pharmacists can provide a medication review service in the form of a MedsCheck ${ }^{\text {ii }}$ within the community pharmacy setting, research exploring the impact of this service on medication adherence is limited. ${ }^{28-31}$

To our knowledge, research exploring the impact of a community pharmacist-delivered medication or healthrelated service specifically for people living with SPMI is scarce. A Canadian study demonstrated that pharmacy staff supported mental health consumers by providing follow-up care which focused on medication management and improving health service navigation and most study participants were using multiple psychotropic medications, few were living with SPMI $(\mathrm{n}=36 ; 17.9 \%) .{ }^{32} 33$ Additionally, results from an Australian community-based, pharmacist-led medication support service for participants living with depression and anxiety were positive with significant improvements in relation to participants illness perceptions, medication satisfaction and medication adherence. ${ }^{6}$ Our proposed Randomised Controlled Trial (RCT) builds and expands on this evidence to test the effectiveness of a person-centred, goal-oriented and flexible, pharmacist-led support service for people living with SPMI, with a specific focus on improving medication adherence and managing physical comorbidities. For the purpose of this RCT, SPMI refers to any mental illness that has a continuous and significant effect on a person's daily life, and includes (but is not limited to) the following: schizophrenia and other psychotic disorders, severe and recurrent depression and bipolar disorder.

\footnotetext{
${ }^{i i}$ A MedsCheck is an Australian remunerated service which involves a community pharmacist reviewing a person's medication, identifying any MRPs, and providing related medication information. Australian residents eligible for a MedsCheck service include consumers who are at a higher risk of MRPs, such as those using more than five prescription medicines. ${ }^{28}$
} 


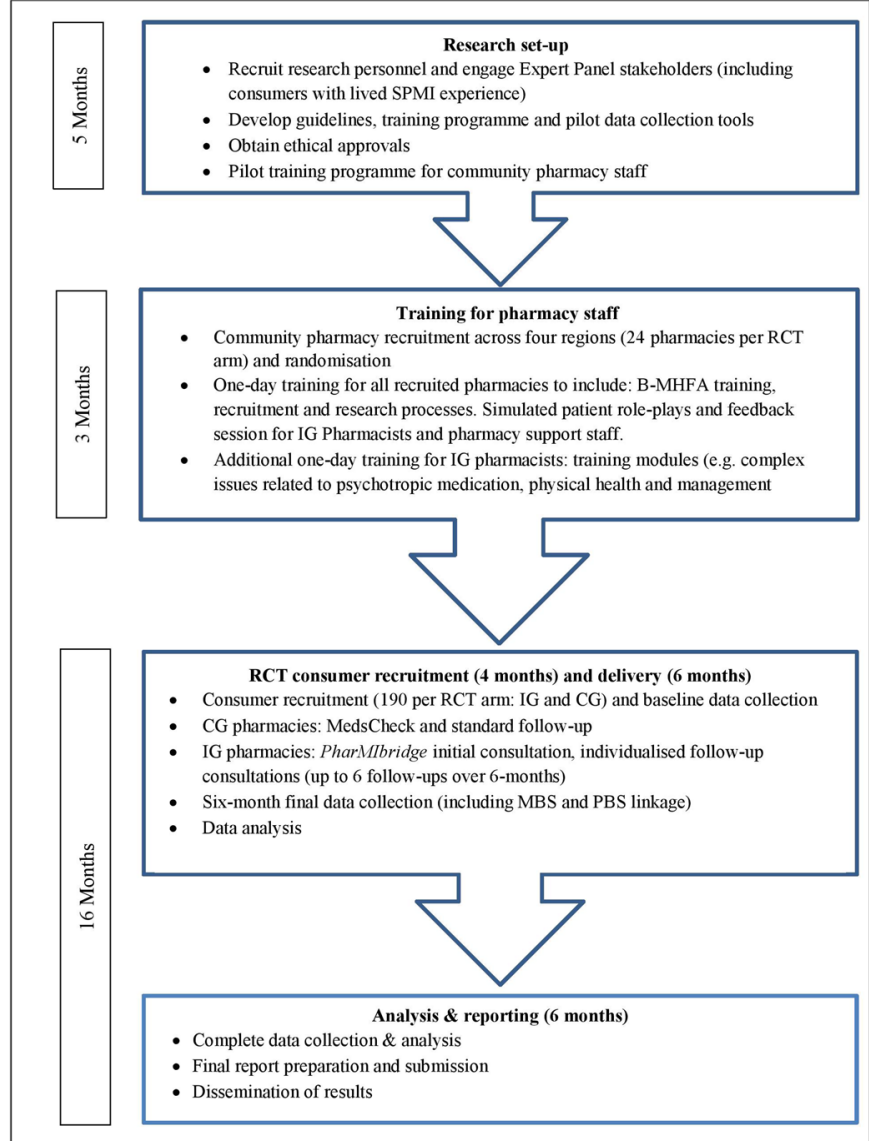

Figure 1 Overview of the research stages and timing. BMHFA, Blended Mental Health First Aid; CG, Comparator Group; IG, Intervention Group; MBS, Medical Benefits Scheme; PBS, Pharmaceutical Benefits Scheme; RCT, Randomised Controlled Trial; SPMI, severe and persistent mental illness.

\section{Study objectives}

The aim of this RCT is to evaluate the effectiveness of 'Bridging the Gap between Physical and Mental Illness in Community Pharmacy (PharMIbridge)'; an intervention comprising an individualised, pharmacist led, support service for people living with SPMI, compared with standard care involving a one-off medication management service (MedsCheck). The primary objective is to test the impact of the PharMIbridge intervention (Intervention Group; IG) on psychotropic medication adherence. It is hypothesised that the PharMIbridge intervention will demonstrate a $20 \%$ increase in adherence at 6-months compared with standard care (Comparator Group; CG). Overall, the objectives of the PharMIbridge RCT are to assess changes in:

- Medication adherence (for psychotropic and other prescribed medication).

- Health outcomes including quality of life, physical health and psychological well-being.

- Community pharmacists' confidence, knowledge and ability to address the medication-related and physical healthcare needs of people living with SPMI.
- Healthcare service acceptability, utilisation and cost-effectiveness.

The findings will be used to inform a more widespread implementation of person-centred service models for people living with SPMI within the primary healthcare sector.

\section{METHODS}

Using a cluster RCT design, community pharmacies will be randomised to deliver either:

1. The PharMIbridge intervention.

2. Standard care involving a one-off MedsCheck service.

Figure 1 provides an overview of the research design, which involves a partnership between two University institutions (Griffith University and The University of Sydney) and two professional pharmacy organisations (The Pharmacy Guild of Australia and The Pharmaceutical Society of Australia). Genuine engagement throughout the entire RCT with individuals with lived experiences of SPMI is critical to success. Given that those living with SPMI require a multifaceted approach to meet their mental and physical healthcare needs, a multidisciplinary team is also a key component of the PharMIbridge RCT. An Expert Panel will be formed, with invitations extended to consumer representatives living with SPMI, and representatives from key organisations such as Mental Health Australia, National Mental Health Consumer and Carer Forum, Sane Australia, the Royal Australian and New Zealand College of Psychiatrists and the Royal Australian College of General Practitioners. The Expert Panel will provide the research team with guidance on the implementation of the RCT, including advice on consumer and healthcare professional engagement, training materials and other resources, and research translation. The research team will work with local primary health networks $(\mathrm{PHNs})^{\text {iii }}$ across RCT sites to recruit eligible community pharmacies into the PharMIbridge RCT.

\section{Study setting and population}

This RCT will be conducted in four Australian regions including urban, rural and remote. Two regions in New South Wales (NSW) (ie, Hunter New England and Northern Sydney), one region in Victoria (ie, Gippsland), and the Australian Capital Territory (ACT) will be included. RCT sites will include a mix of community pharmacies within varied Pharmacy Accessibility/ Remoteness Index of Australia (PhARIA) classification locations, ${ }^{34}$ while also ensuring pharmacies ability to meet

\footnotetext{
${ }^{i i i}$ PHNs are independent organisations funded by the Australian Government. They work closely with local hospital networks and each PHN is overseen by a board of medical professionals. PHNs conduct assessments of the health needs of their region to identify groups of people who need more resources, programmes and services. The information helps PHNs to tailor health services to meet the community's needs (https:// www.healthdirect.gov.au/primary-health-networks-phns).
} 
the eligibility criteria and recruit the required number of consumer participants.

\section{Community pharmacies}

Community pharmacies located within the selected RCT regions will be eligible if they have:

i. Approval to dispense pharmaceutical benefits as part of the Pharmaceutical Benefits Scheme (PBS).

ii. Accreditation for Quality Care Pharmacy standards (quality assurance programme for community pharmacies).

iii. A clientele demographic that includes people with SPMI.

iv. Routinely provided MedsCheck as an established professional service.

v. Guaranteed that the PharMIbridge intervention will be carried out by a registered pharmacist who has completed the training.

vi. A private room/area for confidential conversations and assessments.

vii. Pharmacy owner/manager consent to participate.

viii. An established working relationship with local general practitioner (GP) clinics or centres, mental healthcare teams and/or are already engaged with mental health promotion.

Pharmacies will be randomised into the IG or CG after stratification by region and geographical location (urban/rural/remote). Within each stratum, pharmacies will be randomly allocated in a ratio of $1: 1$ to the IG or CG. Randomisation will occur after all pharmacies for that RCT region have been recruited. The number of full-time equivalent employees will be ranked within each stratum, and pharmacies will be formed into pairs. A list of computer-generated random numbers will be used to allocate either the PharMIbridge intervention (IG) or MedsCheck service (CG) to the first of each pair; the second in each pair will be allocated the alternative service. It is expected that 12 pharmacies will be located in urban, nine in regional/rural and three in remote areas in each of the RCT arms.

\section{Consumer participants}

Trained pharmacy staff (CG and IG) will identify ten eligible consumers per pharmacy for an enrolment period of 6-months. Consumers can also self-identify into the RCT via research promotion or be referred directly from a treating healthcare professional, non-government organisation or mental health service. Eligible consumers need to have capacity to provide informed consent, as assessed by trained pharmacy staff using capacity assessment principles for RCT participation, and consent to pharmacists contacting their treating healthcare team (eg, GP, case manager, psychiatrist). ${ }^{35}$ Furthermore, eligible consumers need to provide consent to allow the researchers to access their associated Medicare Benefits Schedule (MBS), PBS, hospitalisation and emergency department records for a 12-month pretrial and 6-month post-trial period. Specifically, eligibility will require participants to:

i. Be 16 years and over and residing in the community.

ii. Be using at least one antipsychotic or mood stabiliser for at least 6-months continuously, for the treatment of SPMI.

iii. Have a complex medication need (eg, adherence concerns) or unmanaged physical health problems.

Although participants need 6-months of dispensing data for a psychotropic medicine prior to RCT enrolment, medication changes during this time are reasonable, as well as the use of other medication(s) for their mental and physical health. Exclusion criteria include:

i. Aged under 16 years.

ii. Unstable mental health symptoms or deemed as an unsuitable RCT participant by their treating healthcare professionals, pharmacist or carer.

iii. 'When required' or inconsistent use of antipsychotic and/or mood stabiliser medication(s).

iv. Using an antipsychotic or mood stabiliser medication for treatment of an illness not related to SPMI (eg, off-label use of quetiapine for sleep or anxiety).

v. Not consenting to participate to the researchers contacting their treating healthcare practitioner, or to accessing PBS, MBS and hospitalisation data.

vi. Receiving a MedsCheck or Home Medicines Review $(\mathrm{HMR})^{\mathrm{iv}}$ within 12 months prior to RCT enrolment.

\section{PharMlbridge intervention}

An overview of the proposed PharMIbridge RCT is illustrated in figure 2. The intervention will be undertaken by trained community pharmacists randomised into the IG. In addition to the standard care available in all community pharmacies, the intervention will provide IG consumer participants with an in-depth service that is goal oriented, flexible and individualised. Goals will be guided by an in-depth medication review, various self-assessment questionnaires and tools (documented during the Initial Health Review consultation) and be tailored to meet individual needs. There will be a specific focus on overcoming barriers to medication adherence and addressing physical health concerns (table 1). RCT questionnaires and tools are reported under Study outcomes.

IG consumer participants will receive individualised care and support over a 6 -month period (figure 2) to promote medication adherence, optimise the management of their mental illness and encourage lifestyle and pharmacological strategies to improve physical health, with a particular focus on reducing cardiometabolic risk. During the initial consultation (figure 2), IG pharmacists will use motivational interviewing techniques to determine participants' key concerns regarding their medication, adherence and

\footnotetext{
${ }^{\text {iv }}$ A Home Medicines Review (HMR) is a service conducted by an accredited pharmacist in a person's home. The pharmacist reviews the medicines being used by the individual to enhance the quality use of medicines and reduce adverse medicine events.
} 


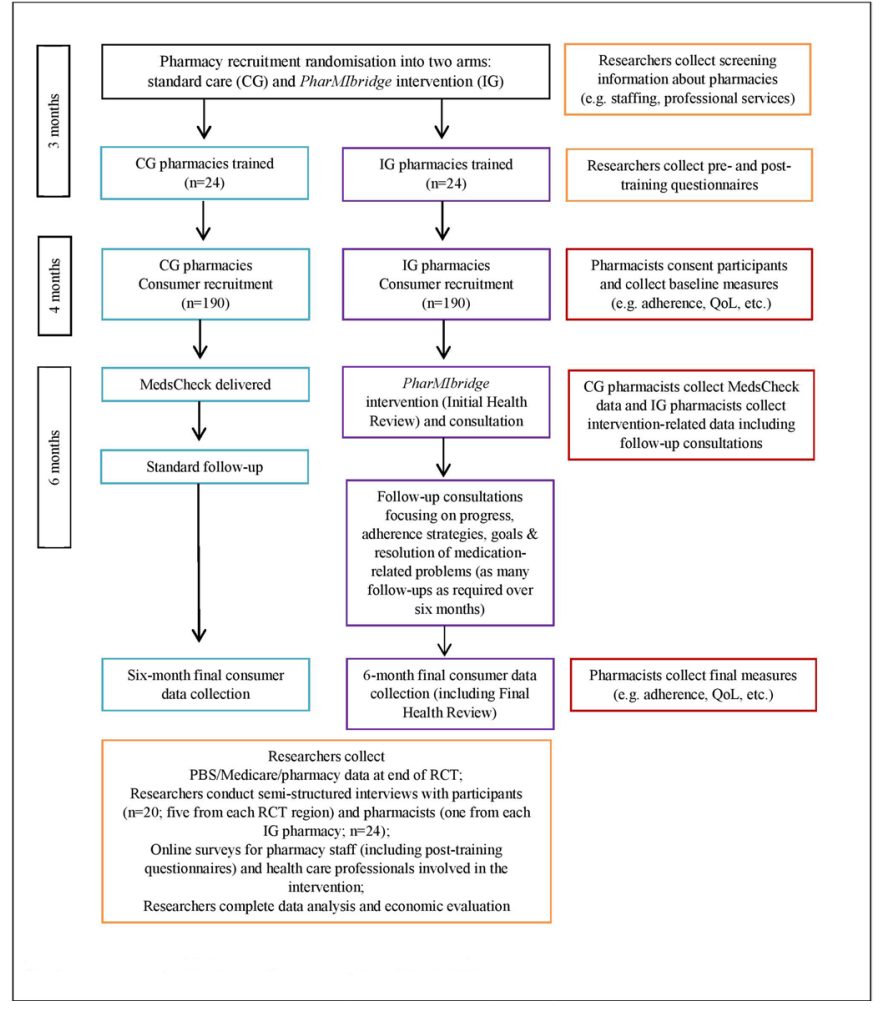

Figure 2 Overview of the PharMIbridge RCT. CG, Gomparator Group; IG, Intervention Group; PBS, Pharmaceutical Benefits Scheme; RCT, Randomised Controlled Trial; QoL, quality of life.

physical and psychological well-being. On identification of three to five key issues, IG pharmacists will: (1) engage participants in a goal-setting discussion and explore strategies to achieve these goals; (2) complete a signed agreement of the support plan and (3) confirm participant's consent to discuss this plan with their nominated healthcare professional (eg, GP, psychiatrist, case manager, etc). The pharmacist will continue to work with the participant over the 6-month intervention period towards goal achievement, with follow-ups as identified with the participant and in their preferred modality (face to face, email, telephone, text messaging). At a minimum, it is expected that participants will have at least three face-to-face consultations with IG pharmacists (including the Initial and Final Health Review consultations). However, additional consultations can occur during the RCT period and participants can obtain pharmacist advice at any time, as needed. At the final 6-month follow-up the IG pharmacist and participant will complete the Final Health Review.

IG pharmacists will be required to keep accurate records of interactions and outcomes using a cloud-based software platform (GuildCare NG), which is currently used in many community pharmacies when delivering professional services. Support plans, medication-related reports and follow-up consultations, recorded as part of the PharMIbridge intervention, will be accessible to a participant's nominated healthcare professional for their direct input, through GuildCare NG. IG pharmacists will work collaboratively with participants' nominated healthcare practitioner(s), updating them regularly via the Guildcare platform and usual communication mechanisms especially when there are any concerns needing rapid intervention.

\section{Comparator arm}

Participants recruited by a CG pharmacy will receive a standard MedsCheck service after enrolment and then continue to receive the usual care that the community pharmacy provides. All requirements outlined for the MedsCheck service will be followed, noting that some participants may not have otherwise been eligible for this government funded service (eg, not taking five or more medicines). Pharmacists will schedule a follow-up consultation to complete final RCT outcome assessments with participants 6-months following recruitment into the RCT.

\section{Training}

Previous evidence-based programmes will inform the development of the education and training materials for the RCT. This includes the training modules used in the Australian mental health feasibility study, ${ }^{36}$ as well as research into pharmacists' knowledge and mental health literacy, ${ }^{37}$ mental health stigma, ${ }^{13} 38$ Mental Health First $\operatorname{Aid}^{\mathrm{v}}$ (MHFA) ${ }^{39}$ simulated patient assessments, ${ }^{40}{ }^{41}$ consumer-led education, ${ }^{42}$ consumer perspectives and consumer/ carer trainers. ${ }^{43-45}$ A training working group will oversee and develop the training content required to deliver the intervention. This group will comprise representation from content experts, consumer representatives and the partner organisations. Face and content validity of all newly developed training material will be ensured through collaborations with mental health consumers, the multidisciplinary Expert Panel and professional pharmacy and mental health organisations. Training will be delivered in such a way that it facilitates a naturalistic approach to enable pharmacists to use their discretion to individually tailor the PharMIbridge intervention for each participant.

Training will involve a 1 or 2-day programme depending on RCT arm allocation, with IG pharmacists attending both training days:

- First training day.

Pharmacy staff in both IG and CG arms will complete Blended MHFA for Pharmacy (B-MHFAP) ${ }^{\mathrm{vi}}$ training, involving a 6-hour online module completed prior to attending the half-day face-to-face training component. ${ }^{46}$ It will be delivered by an accredited instructor and is

\footnotetext{
${ }^{\mathrm{v}}$ Mental Health First Aid (MHFA) courses teach people simple, practical first aid skills to help anyone experiencing mental health problems, even if they are experiencing a crisis. A standard course is usually delivered as a 2-day workshop by accredited instructors. Blended MHFA is delivered in blended mode combining online learning and a half-day face-to-face workshop. (https://mhfa.com.au/take-course).

${ }^{\text {vi }}$ The blended MHFA in the Pharmacy (B-MHFAP) course uses case-studies, videos and resources tailored to pharmacy staff learning needs and is completed in a blended format, combining eLearning and a follow-up 4 hour face-to-face session. https:// mhfa.com.au/courses/public/types/blendedpharmacy.
} 
Table 1 Data collection

Time point in weeks (RCT)

\begin{tabular}{|c|c|c|c|c|c|}
\hline Tyne of evaluation & Descrintion/measure & Data source & Before & 0 & 26 \\
\hline & MPR and medicine persistence & PBS data & & & \\
\hline & $\mathrm{RAM}^{49}$ scale & $\begin{array}{l}\text { GuildCare NG documentation } \\
\text { (all consumer participants) }\end{array}$ & & & \\
\hline Goal achievement & $\begin{array}{l}\text { Type and number of goals set, goal } \\
\text { achievement (GAS) }\end{array}$ & $\begin{array}{l}\text { GuildCare NG documentation } \\
\text { (IG consumer participants) }\end{array}$ & & & \\
\hline Clinical measurements & $\begin{array}{l}\text { Clinical measures for each consumer } \\
\text { participant } \\
\text { Weight/height (body mass index) } \\
\text { Abdominal girth } \\
\text { Blood pressure }\end{array}$ & $\begin{array}{l}\text { GuildCare NG documentation } \\
\text { (all consumer participants) }\end{array}$ & & & \\
\hline \multirow[t]{2}{*}{ Impact } & $\begin{array}{l}\text { Consumer reported experience measure } \\
\text { Semistructured interviews (service } \\
\text { implementation, acceptability) } \\
\text { Online survey (service acceptability) }\end{array}$ & $\begin{array}{l}\text { IG consumer participants only } \\
\text { IG consumer participants ( } \mathrm{n}=20) \text {, } \\
\text { IG pharmacists }(\mathrm{n}=24) \\
\text { Other healthcare professionals } \\
\text { (eg, GP, psychiatrist, MH case } \\
\text { manager) and IG pharmacy staff }\end{array}$ & & & \\
\hline & Healthcare utilisation & Primary care, MBS, hospitalisation & & & \\
\hline Economic appraisal & $\begin{array}{l}\text { RCT-based economic evaluation (eg, resources, } \\
\text { incentive payments, time) }\end{array}$ & $\begin{array}{l}\text { Cost of intervention, MBS, PBS, } \\
\text { pharmacy dispensing data }\end{array}$ & & & \\
\hline
\end{tabular}

Hospitalisation, emergency department admissions, MBS and PBS data will be obtained for 12 months before, and the entire duration of the RCT. Health outcomes data and prescription dispensing data will be obtained for the entire length of the RCT.

AQoL-6D, Assessmentof Quality of Life; ASSIST, Alcohol Smoking and Substance Involvement Screening Test; BIPQ, Brief IIIness Perception Questionnaire; BPI, Brief Pain Inventory; DAI-10, Drug Attitude Inventory 10; DOCUMENT, Drug selection, Over/Under dose, Compliance, Untreated indications, Monitoring, Education orinformation, Non-clinical, Toxicity or adverse reactions; GAS, Goal Attainment Scale; ISI, Insomnia Severity Index; K6, Kessler Psychological Distress Scale; MPR, Medicine PossessionRatio; M3Q, My Medicines and Me Questionnaire; MTBQ, Multimorbidity Treatment Burden Questionnaire; PAVS, Physical Activity Vital Sign Questionnaire; PSQI, Pittsburgh Sleep Quality Index; RAM, Reported Adherence to Medicine Scale; SIMPAQ, Simple Physical Activity Questionnaire.

certified for 24 Continuing Professional Development points. Training on research processes, including participant recruitment and consent, service delivery and use of GuildCare NG as a platform for all data collection, will be delivered to both IG and CG pharmacy staff in separate groups to ensure they are 'research-ready.' Established guidelines for providing MedsCheck services will be utilised by CG pharmacists. ${ }^{47} \mathrm{~A}$ standard operating procedure will be provided to all pharmacists outlining the various steps in the research process.

The remainder of the first day of training for IG pharmacy staff will involve the use of simulated patient roleplays with the accredited B-MHFAP instructors and mental health consumers to encourage staff to practice their newly acquired MHFA skills. ${ }^{40} 418$ Simulated patient roleplays with on-the-spot performance feedback will allow pharmacy staff to demonstrate their newly acquired skills in a safe, authentic and supportive learning environment. Research has highlighted the importance of behavioural assessments post-training due to the potential for over and/or under, estimating skills through self-reported measures. ${ }^{40}$ Research has also indicated that both participants and observers of simulated B-MHFAP role-plays report higher self-reported confidence than those who receive MHFA only, and that maintain improvements in confidence post-training. ${ }^{41}$ 
- Second training day.

IG pharmacists will be required to complete four interactive training modules on key issues associated with psychotropic medication use and physical health, and how to address these issues for people living with SPMI, using evidence-based tools. The modules will be developed by the training working group and led by experts (including psychiatrists, psychopharmacologists and consumer consultants) and will include the following topics:

1. Complex issues relating to psychotropic medication (adherence and side effects). This module will discuss the role of psychotropic medications, including an exploration of the barriers and enablers to adherence, and the consequences of poor adherence. It will also discuss complex issues relating to psychotropic medication use, such as weight gain and diabetes.

2. Physical health issues for people living with SPMI. This module will explore the evidence regarding the importance of physical healthcare and exercise for people living with SPMI. It will also include information on active lifestyles, cardiometabolic risk, tobacco use and resources.

3. Evidence-based strategies to improve medication adherence and physical healthcare. This module will equip pharmacists with evidence-based strategies including guidance on addressing cardiovascular risk factors and sensitive issues such as weight management, smoking cessation, dental health and medication adherence.

4. Communication, motivational interviewing and goal setting. This module will help pharmacists develop appropriate and person-centred communication skills and strategies to work with participants to set, and reach, their mental and physical health goals.

Case studies and role-play activities will be integrated throughout the modules to provide IG pharmacists with opportunities to practice and apply their newly acquired skills, including the use of research tools and questionnaires. Prior to the first workshop commencing specific aspects of the training will be video-recorded to consistency in delivering the intervention to all workshops. The training modules will be made available online for IG pharmacists to access as needed for reference purposes.

The continued well-being of participants living with SPMI is a key aspect of the training. Information on mental health and crisis support numbers and websites will be part of the resources provided, for example, Lifeline, BeyondBlue. Furthermore, communication between pharmacists and the participant's treating team will be ongoing throughout the RCT, thus helping to ensure that any deterioration in health status, or concerns about well-being, will be immediately recognised and addressed.

Recruited community pharmacies will be required to send at least two pharmacists (one of whom can be an intern pharmacist ${ }^{\mathrm{vii}}$ working under the supervision of a registered pharmacist) to complete the PharMIbridge training components. All pharmacies will be encouraged to invite additional pharmacy support staff to complete and attend the B-MHFAP component and information session on research processes, to support pharmacists in delivering the intervention/comparator and establish 'Mental Health Friendly Pharmacies'. Ultimately, trained registered pharmacists will take responsibility for delivering the PharMIbridge intervention. For example, while only the pharmacist can be involved in addressing MRPs, a pharmacy support staff member with well-established consumer relationships could assist with promotion, be involved with identifying eligible participants, making follow-up appointments or sending reminders.

A pilot workshop of the non-B-MHFAP training components will involve a convenience sample of pharmacy students, intern and registered pharmacists, and all RCT trainers/mentors. Feedback will be sought through pretraining and post-training questionnaires and focus group discussions at the conclusion of pilot training. This feedback will help identify whether modifications to the training are required prior to its delivery as part of the RCT.

\section{Training mentors}

The IG training components will be delivered by a local pharmacist and consumer mentor partnership in each RCT region who will also provide ongoing support for IG pharmacy staff throughout the RCT. The combination of a pharmacist educator with experience in community pharmacy practice and a consumer educator with lived experience of mental illness working as a mentor pair has been shown to facilitate implementation of a mental health support service for consumers with depression and anxiety. ${ }^{43}$ The mentor pair will personally visit the IG pharmacies in their RCT region on a minimum of two occasions during the intervention period. In addition to these visits, mentors will provide regular support through phone calls, emails and other modalities, as required. Moreover, the training team will provide two refresher webinars in each RCT region; these will be led by the two mentors. Webinars will focus on problemsolving discussions and co-learning perhaps using examples of 'what has worked well' / 'not so well' to stimulate conversation. Refresher content will be based on two additional, prerecorded, case scenarios allowing pharmacists to revise the skills they have been applying in practice.

\footnotetext{
${ }^{\text {vii }}$ Pharmacy interns are graduates of an approved study programme completing a period of supervised practice (internship). They hold provisional registration and once the internship is completed and they pass the registration examination they are eligible to apply for general registration (www.pharmacyboard.gov.au/Registration/Internships.aspx).
} 


\section{Study outcomes}

Primary outcome

The primary outcome is the difference in medication adherence rates between the IG and CG at RCT conclusion (6-months). Medication adherence will be identified using both routinely collected data (pharmacy dispensing and PBS) and self-reported measurements of adherence; the Reported Adherence to Medicine ${ }^{49}$ scale which has been validated for use among people with psychosis, schizoaffective disorder and schizophrenia. ${ }^{50}$ PBS and pharmacy dispensing data will be used to confirm self-reported data by calculating: (1) the medicine possession ratio, which refers to the proportion of days that a consumer is in possession of their medicine within a defined period; and (2) Medicine Persistence, which refers to a specified time period when there has been a delay in medicine re-fill. ${ }^{51} \mathrm{~A}$ pilot of the selected tools will be undertaken in the set-up stage of the PharMIbridge RCT (figure 1 and table 1) to ensure that they are practical, valid and feasible to use within the RCT time frame.

\section{Secondary outcomes}

\section{Health outcomes}

Clinical measures, including weight, body mass index, abdominal girth and blood pressure, ${ }^{52}$ will be collected at two time points; when participants complete the baseline and 6-month follow-up questionnaires. Pharmacists will collect these measures in the pharmacy and/or from patient/healthcare records. Additionally, participants will be asked (at baseline and 6-month follow-up) if, in the last 12 months, they have had a risk assessment for cardiovascular disease and a glycated haemoglobin blood test to assess for diabetes. Participants physical health will be evaluated using self-reported questionnaires, including the Physical Activity 'Vital Sign' Questionnaire ${ }^{53}$ and selected questions from the Australian Health Survey on Dietary Behaviour. ${ }^{54}$ The Alcohol, Smoking and Substance Involvement Screening Test will also be used to screen for substance use. ${ }^{556}$

Additional tools such as the My Medicines and Me Questionnaire $^{57}$ exploring psychotropic medication side effects; the Simple Physical Activity Questionnaire ${ }^{58} 59$ which identifies time spent on physical activity; the Pittsburgh Sleep Quality Index ${ }^{60}$ exploring sleep quality, the Fagerström Test for Nicotine Dependence ${ }^{61}$ and the Brief Pain Inventory ${ }^{62}$ identifying pain-related concerns, will assist IG pharmacists with intervention delivery. ${ }^{62} 63$ This combined information will inform the development of goals for IG consumer participants, with goal achievement reviewed at each follow-up, and at the final 6-months, consultation using the Goal Attainment Scale. ${ }^{64}$

\section{Quality of life, medication experiences and illness beliefs}

Difference in self-reported quality of life (eg, Assessment of Quality of Life-6D), ${ }^{65}$ psychological well-being (Kessler Psychological Distress Scale), sleep problems (Insomnia Severity Index) ${ }^{66}$ medication burden (Multimorbidity Treatment Burden Questionnaire), ${ }^{67}$ attitudes (Drug
Attitude Inventory 10), ${ }^{68} 69$ illness beliefs (Brief Illness Perception Questionnaire, BIPQ), ${ }^{70}$ and healthcare service utilisation (eg, frequency of GP follow-up or referrals, pharmacist action/s, hospitalisation(s) and emergency department admissions) will be measured between and within RCT arms at baseline and at 6-months. An evaluation of the identification and number and type of MRPs (including side-effects), and adherence to other prescribed medication, will be undertaken at baseline and 6-month follow-up.

It is anticipated that the PharMIbridge intervention will further optimise healthcare utilisation by increasing pharmacist communication and collaboration with other health practitioners and other support people involved in the participant's care. The RCT will involve increased use of MedsChecks by community pharmacists for this vulnerable population. Difference in Federal Government healthcare resource use (measured as the total of primary healthcare services, laboratory tests and medications; and MBS data) will be sought from the Australian Government Department of Human Services, with participant consent.

\section{Pharmacy staff evaluation of training}

Evaluation of the B-MHFAP training will be undertaken as a standard component of this accredited course. ${ }^{71}$ An evaluation of the PharMIbridge training programme in terms of its effectiveness (in comparison to B-MHFA alone) to reduce stigma and build the required skills for pharmacists to work with people living with SPMI will involve pretraining and post-training questionnaires, which will be repeated at RCT conclusion. The questionnaires will collect both qualitative and quantitative data including items adapted from validated scales such as the Social Distance Scale, ${ }^{72}$ the Mental Illness Attitude Questionnaire $^{73}$ and the Metabolic-Barriers, Attitudes, Confidence, and Knowledge Questionnaire. ${ }^{74}$ Additional questions will specifically focus on pharmacist confidence in providing MHFA, ${ }^{41}$ willingness to provide services for mental health consumers ${ }^{13} 7576$ and barriers and incentives to working with people living with SPMI. ${ }^{6}$

\section{PharMlbridge service evaluation}

A proportion of IG consumer participants and pharmacists will be invited to participate in a telephone interview at RCT conclusion to qualitatively explore the acceptability and impact of the PharMIbridge intervention. Up to five consumer participants from each RCT region will be invited for interviews $(\max =20)$ as well as one pharmacist from each pharmacy who participated in the RCT $(\max =24)$. The participant interview guide will explore topics including: (1) medication adherence and current medications; (2) types of MRPs experienced and resolved; (3) health literacy in relation to psychotropic medicines; (4) changes in relationship between participants, pharmacists and other healthcare professionals and (5) satisfaction with the PharMIbridge intervention. An additional participant-reported experience measure 
will be developed and included at the end of the service delivery in the Final Health Review. A short online survey will also be developed to explore service acceptability for other health practitioners who interact with the intervention, and other pharmacy staff, for example, pharmacy assistants.

\section{Data collection}

The RCT data collection tools and processes will be piloted prior to use (figure 1). Data will be collected from consumer participants, pharmacy staff and other healthcare professionals (table 1 ).

Consumer participants will be required to consent to their MBS, PBS, hospitalisation and emergency department data being accessed by researchers for a period of 12 months prior to, and 6-months after, enrolment in the RCT. The reason for collecting data about resource use up to 12 months prior to the RCT is to test for baseline differences which can then be adjusted for during analysis.

Participant data collected by pharmacists will be entered directly into purpose-built IG and CG research modules within GuildCare NG. This cloud-based pharmacy software platform is used by the majority of community pharmacies in Australia for the delivery of professional service programmes such as MedsCheck; it also links to dispensing systems. In the IG, nominated members of the participant's treating team (ie, GP, psychiatrist, mental health case manager) will be provided with access to their individual consumer participant's research records to facilitate timely access to, and exchange of, shared information.

Participating community pharmacies will receive funding to support their research involvement, including an administration fee for training attendance, research administration and data management (ranging from US $\$ 3700-U S \$ 10712$ depending on urban/rural/remote location), as well as a per-participant fee for initial and follow-up consultations, and for 6-month data collection. IG pharmacies will be remunerated for the baseline data collection and Initial Health Review (75 min; US\$167), follow-up consultations (Level 1: up to three consultations; US\$66, or level 2: greater than three consultations; US\$133), and for the Final Health Review and follow-up data collection (30 min; US\$167). Service fees for CG pharmacists include the MedsCheck consultation and baseline data collection (60 min; US\$133) and the 6 -month follow-up data collection (30 min; US\$66). A gift voucher valued at $\$ \mathrm{~A} 30$ will be offered to all consumer participants at the final, 6-month data collection point in recognition of their time for completing data collection processes that would not normally be part of their healthcare.

\section{Sample size}

Based on a recent study conducted in a similar population, ${ }^{6}$ we assume the baseline rate of non-adherence assessed using pharmacy dispensing data will be $56 \%$.
This outcome will be available for all participants (unless actively withdrawn), regardless of treatment adherence. We assume the percentage of non-adherent participants in the CG arm will drop to $51 \%$ (based on the results of previous studies exploring adherence as an outcome of a MedsCheck service) $)^{28-31}$ and the percentage of nonadherent participants in the IG arm will drop to $35 \%{ }^{6}$ With alpha $=0.05$, the RCT will have $80 \%$ power to find a statistically significant difference between RCT arms with 149 individuals recruited to each arm (without accounting for clustering).

As this is a cluster RCT, we need to account for the nonindependence of observations from individual participants recruited from the same pharmacy. We assume a median number of 10 participants at each pharmacy, and conservatively assume the intraclass correlation coefficient $(\mathrm{ICC})=0.03$, giving a design effect of 1.27 . Consequently, a total of 190 participants will need to be recruited in each arm (380 in total). To achieve this, 10 consumer participants will need to be enrolled from each of 38 pharmacies. Assuming that $20 \%$ of pharmacies will withdraw from the RCT without enrolling any participants, a total of 48 pharmacies will need to be recruited.

\section{Data analyses}

Descriptive statistics will be used; mean (SD) or median (25-75th percentile) for continuous data and frequency (percentage) for categorical data. We will compare the primary outcome of percentage adherence between RCT arms using a mixed-effects logistic regression model, with treatment arm entered as the fixed effect and pharmacy entered as a random effect, to account for possible nonindependence of observations from participants who attend the same pharmacy. Results will be reported as odds ratios and $95 \%$ Wald-based CIs.

For secondary analyses, interval data will be analysed using mixed-effects linear regression, binary data using mixed-effects logistic regression, and count data using mixed-effects Poisson regression models. In all cases, the likely non-independence of data from individuals enrolled through the same pharmacy will be accounted for as random effects in the regression models. Analyses will be based on the intention-to-treat principle, with participants analysed according to the treatment they were allocated to receive. Data will be analysed using Stata statistical software (StataCorp), with significance set at less than 0.05 .

Qualitative interviews will be audio recorded (with participant consent), transcribed verbatim and quality checked. Thematic analysis will be conducted by experienced qualitative researchers within the research team. This process involves reading and rereading transcripts to identify, analyse and report patterns within the data, leading to the identification of similar codes across the entire data set. ${ }^{77}$ Initial codes are thereby sorted into themes and subthemes. $^{77}$ 


\section{Economic evaluation}

We hypothesise that the provision of the PharMIbridge intervention will be cost-effective compared with standard care from a healthcare sector perspective with regard to RCTbased costs and outcomes (cost per additional participant adherent/cost per (proxy) health outcome). The base case analysis will comprise the cost per additional 'adherent' participant for the IG compared with the CG and reported as the incremental cost-effectiveness ratio (ICER). The outcome for the base case analysis is the difference in the change in the proportion of 'adherent' participants from baseline to RCT conclusion, between the IG and CG. Subgroup analyses will be conducted for the primary outcome measure (adherence) based on participant characteristics. Regression analyses using 'adherence' as the dependent variable will be performed to assess if the effect of the intervention varies by SPMI diagnosis, age, gender, pharmacy location and number of other medications taken concurrently. Any evidence of non-linearity will be adjusted for using appropriate transformations.

A cost per quality-adjusted life year (QALY) gain will also be conducted based on health-related quality of life data collected within the trial. QALYs will be estimated by applying Australian utility weights and using the area under the curve approach. Difference in QALYs between the IG and CG will be estimated using linear or non-linear regression methods depending on the nature of the data. The analytical time frame for the cost-effectiveness analyses will be limited to that of the follow-up period of the trial. This is the most conservative approach relative to translation of the primary outcome measure, adherence, where there is likely to be heterogeneity of medications and conditions.

Costs will be considered from the health system perspective and include both the cost of interventions and the cost consequences from potential changes in healthcare utilisation. The difference in health resource use for all participants will be collected and analysed based on administratively collected MBS, PBS and pharmacy dispensing data.

\section{Included costs}

The difference in health resource use for all participants will be collected and analysed from their MBS and PBS records 6-months prior to, and during, enrolment in the RCT. Impact of seasonality will also be assessed for the same 12-month period. The economic evaluation will quantify costs associated with the PharMIbridge intervention at a participant level in the eligible population. The costs measured in the economic evaluation should be equivalent to their 'opportunity cost', that is, assessed in terms of the monetary value of the benefits that are foregone because the resources are not available for their best alternative use. The opportunity cost will be estimated from the market costs of the resources consumed, whenever available. Costs from the RCT include, but will not be limited to: (1) the training of pharmacists to provide the PharMIbridge intervention; (2) the fee-for-service to pharmacists and (3) the clinical software adaption to facilitate the identification of 'non-adherent' participants and record interventions.

\section{Cost data analysis}

The source of any variation (if observed) in costs between intervention and comparator arms, as well as the cost 'drivers', will be investigated with a generalised linear model (GLM) using a standard statistical software package (STATA). As cost data are not likely to be normally distributed, a gamma log-link function or similar will be used, with appropriate testing of the GLM specification using the modified Park test and link test. Full costing will be provided for the RCT. If certain costs are not included in the economic evaluation, any assumptions, and the effect of their exclusion, will be specified in the analysis.

Costing of the PharMIbridge intervention will be undertaken as follows: (1) identify administrative resources used in programme management, with RCT-specific administrative resources separated from activities that would be included in a national roll-out of the programme; (2) some resource use will be measured in natural units (eg, clinical staff time spent on delivering the PharMIbridgeintervention) and will be valued in monetary units; (3) other resources can be directly valued (eg, incentive payments to staff and services) and (4) if the PharMIbridge intervention delivery requires additional equipment (eg, privacy screen), market costs will be estimated with amortisation applied over the useful life of the equipment.

\section{Sensitivity analysis}

The uncertainty in effect sizes and costs will translate into a degree of uncertainty around the estimated ICER. This uncertainty will be explored using a system of seemingly unrelated regression equations. This has been chosen because it has been argued that such methods are generally robust to skewed data, and to allow for any correlation between costs and effects. The cost and adherence (or QALY) regressions will be run simultaneously, including SPMI diagnosis, age, gender, pharmacy location and number of other medications taken concurrently included (as covariates) to explore potential subgroup differences with respect to adherence, costs or cost-effectiveness. Additionally, in order to estimate the level of uncertainty associated with the decision as to whether or not the intervention was cost-effective, we will use the non-parametric bootstrap technique (with 5000 replications sampled with replacement) to estimate the probability that the intervention is cost-effective over a range of willingness to pay per outcome (adherer/QALY) according to the cost-effectiveness acceptability curve.

\section{Financial appraisal}

Financial appraisal and full pathway costing will be undertaken as part of the PharMIbridge RCT. Where assumptions for identification, measurement and valuation of economic costs differ to financial costs, these differences will be specified. The cost to the health sector if the PharMIbridge 
intervention was implemented nationally will be extrapolated over a 5-year time frame.

\section{Patient and public involvement}

To provide maximum benefit to people with SPMI, health services need to be relevant, accessible and responsive to their needs. The views of people with lived experience of a mental illness will be paramount to the success of the PharMIbridge RCT, from their involvement in the development of the RCT protocol as members of the research team, development and implementation of the intervention with pharmacy staff as trainers and mentors, and as representatives on the Expert Panel who will have oversight across the lifetime of the research. A working group of partner organisation representatives, consumer representatives and experts actively involved in improving mental healthcare will codevelop training material. People with lived experience of SPMI will also inform dissemination strategies based on the RCT findings.

\section{DISCUSSION}

The PharMIbridge RCT will use the medication expertise of community pharmacists to support medication adherence and address the physical and mental health needs of participants using psychotropic medication for SPMI. The focus on the physical health of people living with SPMI is of utmost importance given the considerable life expectancy gap attributable to physical comorbidities in this population. Beyond improved consumer participant well-being, the PharMIbridge RCT is expected to facilitate better relationships between pharmacists and local health services, including community mental health teams, PHNs and GPs. Lastly, by providing a diverse range of mental health services and resources and by increasing community outreach to mental health and non-mental health related services IG pharmacies will become more prominent in the mental health sector.

Although the provision of a MedsCheck service for people using high-risk medication such as psychotropics (in addition to other criteria) is a currently funded service, there is evidence that pharmacists' mental health literacy concerning SPMI is lower in comparison to other mental illnesses. ${ }^{37}$ Most people using antipsychotics and/or mood stabilisers would be eligible for a MedsCheck, however, we believe that this vulnerable population may be missing out on this service as it is not specifically targeted towards mental health consumers. Therefore, the proposed PharMIbridge intervention is a novel service within the community pharmacy setting and is, to our knowledge, the first RCT to determine the impact of a pharmacist-led support service for people living with SPMI.

All pharmacy staff involved in the RCT will receive B-MHFA. Hence, the PharMIbridge RCT provides an opportunity for all consumer participants to access a recognised standard level of care. Furthermore, analyses conducted on the CG consumer participant outcomes will allow us to identify the impact of MedsCheck for consumers living with
SPMI. This is particularly important given the current lack of data regarding the impact of MedsChecks on medication adherence. Furthermore, it is unrealistic and unethical to expect that consumer participants living with SPMI and enrolled into the CG receive no form of care or service. These participants will be asked to complete a range of surveys at baseline which are likely to identify adherence and health issues, and consequently pharmacists would be expected, as healthcare professionals, to intervene in such situations. Thus, the research team also anticipates positive outcomes for CG participants, although this effect is hypothesised to be inferior to IG participants.

There are some limitations to this RCT. While a key focus of this research is the physical healthcare needs of consumer participants, the follow-up time of 6-months is insufficient to use physical health as a primary outcome measure. The RCT duration was selected to meet the specified time-frame requirements of the funding body. Hence, the long-term impact of the PharMIbridge intervention cannot be determined. It should be noted that the RCT sites, while specifically chosen to reflect a variety of geographical regions, types of pharmacies, mental health services and socioeconomic factors, may not be generalisable to the Australian population. Lastly, as adherence is known to be an inherently difficult concept to measure, to ensure accurate and comprehensive assessment the research team will use multiple objective and subjective measures to assess this outcome. Notwithstanding these limitations, the PharMIbridge RCT will provide valuable insights into the future implementation of a community pharmacy based, mental health support service across Australia.

\section{Ethics and dissemination}

This RCT protocol and training programme has been approved by Griffith University Human Research Ethics Committee (2019/473 and 2019/493). The trial has been registered with the Australia and New Zealand Clinical Trials Registry (ANZCTR12620000577910). At the conclusion of the PharMIbridge RCT, a report will be submitted to the Australian Government Department of Health and partner organisations. A dissemination plan will be developed by the research team, project partners and the Expert Panel. A summary of findings for all participating pharmacies and consumer participants will be provided at the end of the RCT. Results will be published as manuscripts in peer-reviewed journals and presented at national and international scholarly conferences and symposia.

\section{Author affiliations}

${ }^{1}$ Menzies Health Institute Queensland, Griffith University, Brisbane, Queensland, Australia

${ }^{2}$ Faculty of Medical and Health Sciences, Auckland University, Auckland, New Zealand

${ }^{3}$ Sydney Pharmacy School, Faculty of Medicine and Health, The University of Sydney, Sydney, New South Wales, Australia

${ }^{4}$ School of Pharmacy and Pharmacology, Griffith University, Gold Coast, Queensland, Australia 
Twitter Amanda J Wheeler @AmandaJWheeler1, Claire L 0'Reilly @oreilly_claire, Sarira El-Den@SariraEl_Den, Joshua Byrnes @jbhealthecon and Sara S McMillan @SaraSinclairMcM

Acknowledgements This RCT was developed in partnership with The Pharmacy Guild of Australia, the Pharmaceutical Society of Australia, Griffith University and The University of Sydney. We thank Dr Helen Stapleton for her feedback on the manuscript.

Contributors AJW, CLO, SSM and SE-D designed the research protocol and were named investigators on the funding proposal. AJW and CLO are coprincipal investigators and led the intervention and training development and evaluation plan. JB provided expert oversight of the economic evaluation plan, assisted with the selection of primary outcome measures and datasets. SSM and SE-D led the ethics application process, review of data collection tools and contributed to the intervention and training development and evaluation plan. RSW provided statistica expertise and sample size calculations for the RCT. All authors were involved in the revision of this protocol and made a significant contribution towards its intellectual content and approved the final version of the manuscript.

Funding This activity received grant funding from the Australian Government. Competing interests None declared.

Patient and public involvement Patients and/or the public were involved in the design, or conduct, or reporting, or dissemination plans of this research. Refer to the Methods section for further details.

\section{Patient consent for publication Not required.}

Provenance and peer review Not commissioned; peer reviewed for ethical and funding approval prior to submission.

Open access This is an open access article distributed in accordance with the Creative Commons Attribution Non Commercial (CC BY-NC 4.0) license, which permits others to distribute, remix, adapt, build upon this work non-commercially, and license their derivative works on different terms, provided the original work is properly cited, appropriate credit is given, any changes made indicated, and the use is non-commercial. See: http://creativecommons.org/licenses/by-nc/4.0/.

\section{ORCID iDs}

Amanda J Wheeler http://orcid.org/0000-0001-9755-674X

Claire L 0'Reilly http://orcid.org/0000-0001-6416-8150

Sarira El-Den http://orcid.org/0000-0001-7500-3351

Sara S McMillan http://orcid.org/0000-0003-3427-4467

\section{REFERENCES}

1 O'Reilly CL, Wong E, Chen TF. A feasibility study of community pharmacists performing depression screening services. Res Social Adm Pharm 2015;11:364-81.

2 Knox ED, Dopheide JA, Wincor MZ, et al. Depression screening in a university campus pharmacy: a pilot project. Journal of the American Pharmacists Association 2006;46:502-6.

3 Rosser S, Frede S, Conrad WF, et al. Development, implementation, and evaluation of a pharmacist-conducted screening program for depression. J Am Pharm Assoc 2013;53:22-9.

4 Nishtala PS, McLachlan AJ, Bell JS, et al. Psychotropic prescribing in long-term care facilities: impact of medication reviews and educational interventions. Am J Geriatr Psychiatry 2008;16:621-32.

5 Schmidt I, Claesson CB, Westerholm B et al. The impact of regular multidisciplinary team interventions on psychotropic prescribing in Swedish nursing homes. J Am Geriatr Soc 1998;46:77-82.

6 McMillan SS, Kelly F, Hattingh HL, et al. The impact of a personcentred community pharmacy mental health medication support service on consumer outcomes. J Ment Health 2018;27:164-73.

7 Gisev N, Bell JS, O'Reilly CL, et al. An expert panel assessment of comprehensive medication reviews for clients of community mental health teams. Soc Psychiatry Psychiatr Epidemiol 2010;45:1071-9.

8 Bell JS, Aslani P, McLachlan AJ, et al. Mental health case conferences in primary care: content and treatment decision making. Res Social Adm Pharm 2007;3:86-103.

9 Finley PR, Rens HR, Pont JT, et al. Impact of a collaborative pharmacy practice model on the treatment of depression in primary care. Am J Health Syst Pharm 2002;59:1518-26.

10 Gable KN, Stunson MJ. Clinical pharmacist interventions on an assertive community treatment team. Community Ment Health $\mathrm{J}$ 2010;46:351-5.
11 Hansson L, Jormfeldt $H$, Svedberg P, et al. Mental health professionals' attitudes towards people with mental illness: do they differ from attitudes held by people with mental illness? Int J Soc Psychiatry 2013;59:48-54.

12 Rao H, Mahadevappa H, Pillay P, et al. A study of stigmatized attitudes towards people with mental health problems among health professionals. J Psychiatr Ment Health Nurs 2009;16:279-84.

13 O'Reilly CL, Bell JS, Kelly PJ, et al. Exploring the relationship between mental health stigma, knowledge and provision of pharmacy services for consumers with schizophrenia. Res Social Adm Pharm 2015;11:e101-9.

14 Wheeler A, Mey A, Kelly F, et al. Education and training for community pharmacists in mental health practice: how to equip this workforce for the future. J MH Training, Ed and Practice 2014:9:133-44

15 Morgan V, Waterreus A, Jablensky A, et al. People living with psychotic illness 2010. Canberra: Australian Government; National Mental Health Strategy, 2011.

16 Australian Institute of Health and Welfare. Mental health services - In brief. Canberra, Australia: Australian Institute of Health and Welfare, 2019.

17 National Mental Health Commission (NMHC). Equally well: improving the physical health and wellbeing of people living with mental illness in Australia. Sydney, Australia: NMHC, 2016.

18 Ehrlich C, Chester P, Kisely S, et al. Making sense of selfcare practices at the intersection of severe mental illness and physical health-An Australian study. Health Soc Care Community 2018;26:e47-55.

19 Morant N, Azam K, Johnson S, et al. The least worst option: user experiences of antipsychotic medication and lack of involvement in medication decisions in a UK community sample. J Ment Health 2018;27:322-8.

20 Young SJ, Praskova A, Hayward N, et al. Attending to physical health in mental health services in Australia: a qualitative study of service users' experiences and expectations. Health Soc Care Community 2017;25:602-11.

21 Wade M, Tai S, Awenat Y, et al. A systematic review of service-user reasons for adherence and nonadherence to neuroleptic medication in psychosis. Clin Psychol Rev 2017;51:75-95.

22 Masand PS, Roca M, Turner MS, et al. Partial adherence to antipsychotic medication impacts the course of illness in patients with schizophrenia: a review. Prim Care Companion J Clin Psychiatry 2009;11:147-54.

23 Farrelly S, Brown G, Szmukler G, et al. Can the therapeutic relationship predict 18 month outcomes for individuals with psychosis? Psychiatry Res 2014;220:585-91.

24 Bell JS, Whitehead P, Aslani P, et al. Drug-related problems in the community setting. Clin Drug Investig 2006;26:415-25.

25 Rubio-Valera M, Chen TF, O'Reilly CL. New roles for pharmacists in community mental health care: a narrative review. Int $J$ Environ Res Public Health 2014;11:10967-90.

26 Valenstein M, Kavanagh J, Lee T, et al. Using a pharmacy-based intervention to improve antipsychotic adherence among patients with serious mental illness. Schizophr Bull 2011;37:727-36.

27 Hartung D, Low A, Jindai K, et al. Interventions to improve pharmacological adherence among adults with psychotic spectrum disorders and bipolar disorder: a systematic review. Psychosomatics 2017;58:101-12.

28 Bouvy ML, Heerdink ER, Urquhart J, et al. Effect of a pharmacistled intervention on diuretic compliance in heart failure patients: a randomized controlled study. J Card Fail 2003;9:404-11.

29 Messerli M, Blozik E, Vriends N, et al. Impact of a community pharmacist-led medication review on medicines use in patients on polypharmacy--a prospective randomised controlled trial. BMC Health Serv Res 2016;16:145.

30 Community Pharmacy Medicines Management Project Evaluation Team. The MEDMAN study: a randomized controlled trial of community pharmacy-led medicines management for patients with coronary heart disease. Fam Pract 2007;24:189-200.

31 Bernsten C, Björkman I, Caramona M, et al. Improving the wellbeing of elderly patients via community pharmacy-based provision of pharmaceutical care: a multicentre study in seven European countries. Drugs Aging 2001;18:63-77.

32 Murphy AL, Gardner DM, Jacobs LM. Patient care activities by community pharmacists in a capitation funding model mental health and addictions program. BMC Psychiatry 2018;18:192.

33 Haslam L, Gardner DM, Murphy AL. A retrospective analysis of patient care activities in a community pharmacy mental illness and addictions program. Res Social Adm Pharm 2020;16:522-528.

34 Australian Bureau of Statistics. ASGC remoteness and classification: purpose and use. Canberra: ABS, 2003. 
35 New South Wales Government. Capacity toolkit. State of New South Wales: Attorney General's Department of NSW, 2008.

36 Wheeler A, Fowler J, Hattingh L. Using an intervention mapping framework to develop an online mental health continuing education program for pharmacy staff. $J$ Contin Educ Health Prof 2013;33:258-66.

37 O'Reilly CL, Bell JS, Chen TF. Pharmacists' beliefs about treatments and outcomes of mental disorders: a mental health literacy survey. Aust N Z J Psychiatry 2010;44:1089-96.

$38 \mathrm{Knox} \mathrm{K}$, Fejzic J, Mey A, et al. Mental health consumer and caregiver perceptions of stigma in Australian community pharmacies. Int J Soc Psychiatry 2014;60:533-43.

39 O'Reilly CL, Bell JS, Kelly PJ, et al. Impact of mental health first aid training on pharmacy students' knowledge, attitudes and self-reported behaviour: a controlled trial. Aust N Z J Psychiatry 2011;45:549-57.

40 El-Den S, Chen TF, Moles RJ, et al. Assessing mental health first aid skills using simulated patients. Am J Pharm Educ 2018;82:6222.

41 Boukouvalas EA, El-Den S, Chen TF, et al. Confidence and attitudes of pharmacy students towards suicidal crises: patient simulation using people with a lived experience. Soc Psychiatry Psychiatr Epidemiol 2018;53:1185-95.

42 O'Reilly CL, Bell JS, Chen TF. Consumer-led mental health education for pharmacy students. Am J Pharm Educ 2010;74:167.

43 Fowler JL, McConachie B, Hattingh L, et al. Mentoring pharmacy staff to implement a medication support service: an evaluation of process and outcomes. Curr Pharm Teach Learn 2018;10:886-94.

44 Hattingh HL, Knox K, Fejzic J, et al. Privacy and confidentiality: perspectives of mental health consumers and carers in pharmacy settings. Int J Pharm Pract 2015;23:52-60.

45 O'Reilly CL, Bell JS, Chen TF. Mental health consumers and caregivers as instructors for health professional students: a qualitative study. Soc Psychiatry Psychiatr Epidemiol 2012;47:607-13.

46 Mental Health First Aid Australia. Blended MHFA in the pharmacy. Victoria: MHFA Australia, 2019.

47 Pharmaceutical Society of Australia. Guidelines for pharmacists providing MedsCheck and diabetes medscheck services. Deakin West, ACT: Pharmaceutical Society of Australia, 2017.

48 O'Reilly Claire L, Moles Rebekah J, Boukouvalas E, et al. Assessing students' mental health crisis skills via consumers with lived experience: a qualitative evaluation. $J$ Ment Health Train 2019;14:361-71.

49 Horne R, Weinman J, Hankins M. The beliefs about medicines questionnaire: the development and evaluation of a new method for assessing the cognitive representation of medication. Psychol Health 1999:14:1-24.

50 Nguyen T-M-U, La Caze A, Cottrell N. What are validated self-report adherence scales really measuring?: a systematic review. $\mathrm{Br} J$ Clin Pharmacol 2014:77:427-45.

51 Sattler ELP, Lee JS, Perri M. Medication (re)fill adherence measures derived from pharmacy claims data in older Americans: a review of the literature. Drugs Aging 2013;30:383-99.

52 Royal Australian and New Zealand College of Psychiatrists. Keeping body and mind together - improving the physical health and life expectancy of people with serious mental illness. Melbourne, Australia and Wellington, New Zealand: Royal Australian and New Zealand College of Psychiatrists (RANZCP), 2015

53 Vancampfort D, Stubbs B, Probst M, et al. Physical activity as a vital sign in patients with schizophrenia: evidence and clinical recommendations. Schizophr Res 2016:170:336-40.

54 Australian Bureau of Statistics. National health survey 2017-2018 questionnaire, 2018.

55 WHO ASSIST Working Group. The alcohol, smoking and substance involvement screening test (assist): development, reliability and feasibility. Addiction 2002;97:1183-94.
56 Humeniuk R, Ali R, Babor TF, et al. Validation of the alcohol, smoking and substance involvement screening test (assist). Addiction 2008;103:1039-47.

57 Ashoorian D, Davidson R, Rock D, et al. A clinical communication tool for the assessment of psychotropic medication side effects. Psychiatry Res 2015;230:643-57.

58 Rosenbaum S, Ward PB, International Working Group. The simple physical activity questionnaire. Lancet Psychiatry 2016;3:e1

59 SIMPAQ. Simple physical activity questionnaire (SIMPAQ), 2019.

60 Smyth C. The pittsburgh sleep quality index (PSQI), 2012.

61 Heatherton TF, Kozlowski LT, Frecker RC, et al. The Fagerström test for nicotine dependence: a revision of the Fagerström tolerance questionnaire. Br J Addict 1991;86:1119-27.

62 Cleeland CS, Ryan KM. Pain assessment: global use of the brief pain inventory. Ann Acad Med Singapore 1994;23:129-38.

63 Dworkin RH, Turk DC, Farrar JT, et al. Core outcome measures for chronic pain clinical trials: IMMPACT recommendations. Pain 2005;113:9-19.

64 Kiresuk TJ, Sherman RE. Goal attainment scaling: a general method for evaluating comprehensive community mental health programs. Community Ment Health J 1968;4:443-53.

65 Richardson JRJ, Peacock SJ, Hawthorne G, et al. Construction of the descriptive system for the assessment of quality of life AQoL-6D utility instrument. Health Qual Life Outcomes 2012;10:38.

66 Bastien $\mathrm{CH}$, Vallières A, Morin CM. Validation of the insomnia severity index as an outcome measure for insomnia research. Sleep Med 2001;2:297-307.

67 Duncan P, Murphy M, Man M-S, et al. Development and validation of the multimorbidity treatment burden questionnaire (MTBQ). BMJ Open 2018;8:e019413.

68 Hogan TP, Awad AG, Eastwood R. A self-report scale predictive of drug compliance in schizophrenics: reliability and discriminative validity. Psychol Med 1983;13:177-83.

69 Nielsen RE, Lindström E, Nielsen J, et al. DAl-10 is as good as DAl30 in schizophrenia. Eur Neuropsychopharmacol 2012;22:747-50.

70 Broadbent E, Petrie KJ, Main J, et al. The brief illness perception questionnaire. J Psychosom Res 2006;60:631-7.

71 Mental Health First Aid Australia. Blended MHFA in the pharmacy. Victoria: MHFA Australia, 2018.

72 Jorm AF, Oh E. Desire for social distance from people with mental disorders. Aust N Z J Psychiatry 2009;43:183-200.

73 Kobau R, Diiorio C, Chapman D, et al. Attitudes about mental illness and its treatment: validation of a generic scale for public health surveillance of mental illness associated stigma. Community Ment Health J 2010;46:164-76.

74 Watkins A, Rosenbaum S, Ward PB, et al. The validity and reliability characteristics of the M-BACK questionnaire to assess the barriers, attitudes, confidence, and knowledge of mental health staff regarding metabolic health of mental health service users. Front Public Health 2017; 5:321-21.

75 Phokeo V, Sproule B, Raman-Wilms L. Community pharmacists' attitudes toward and professional interactions with users of psychiatric medication. Psychiatr Serv 2004;55:1434-6.

76 Cates ME, Burton AR, Woolley TW. Attitudes of pharmacists toward mental illness and providing pharmaceutical care to the mentally ill. Ann Pharmacother 2005;39:1450-5.

77 Braun V, Clarke V. Using thematic analysis in psychology. Qual Res Psychol 2006;3:77-101.

78 World Health Organisation. Guidelines for the management of physical health conditions in adults with severe mental disorders. Geneva: WHO, 2018.

79 Williams M, Peterson GM, Tenni PC, et al. Document: a system for classifying drug-related problems in community pharmacy. Int J Clin Pharm 2012;34:43-52.

80 Kessler RC, Barker PR, Colpe LJ, et al. Screening for serious mental illness in the general population. Arch Gen Psychiatry 2003;60:184-9. 